PROCEEDINGS OF THE

AMERICAN MATHEMATICAL SOCIETY

Volume 132, Number 11, Pages 3153-3158

S 0002-9939(04)07426-X

Article electronically published on May 12, 2004

\title{
DOUBLE COVERING OF CURVES
}

\author{
EDOARDO BALLICO, CHANGHO KEEM, AND SEUNGSUK PARK
}

(Communicated by Michael Stillman)

\begin{abstract}
Let $C$ be a smooth projective algebraic curve of genus $q$ and $g$ an integer with $g \geq 4 q+5$. For all integers $d \geq g-2 q+1$ we prove the existence of a double covering $f: X \rightarrow C$ with $X$ a smooth curve of genus $g$ and the existence of a degree $d$ morphism $u: X \rightarrow \mathbb{P}^{1}$ that does not factor through $f$. By the Castelnuovo-Severi inequality, the result is sharp (except perhaps the bound $g \geq 4 q+5$ ).
\end{abstract}

\section{INTRODUCTION}

The main aim of this paper is the proof of the following result.

Theorem 1.1. Fix integers $g, q$ and $d$ with $q \geq 0, g \geq 4 q+5$ and $d \geq g-2 q+1$. Let $C$ be a smooth projective curve of genus $q$. Then there exist a double covering $f: X \rightarrow C$ with $X$ a smooth connected curve of genus $g$ and $M \in \operatorname{Pic}^{d}(X)$ with $M$ spanned and such that the morphism $X \rightarrow \mathbb{P}\left(H^{0}(X, M)\right)$ induced by $M$ does not factor through $f$.

If $d \leq g-2 q$, no such $M$ may exist for any genus $g$ double covering of a smooth genus $q$ curve by the Castelnuovo-Severi inequality (see e.g. [Ka]). Hence Theorem 1.1 seems to be quite strong. We discuss here the main differences between Theorem 1.1] and [BK1 Theorem 0.1], which was proved using a quite different method. Theorem 1.1 here has the following strong advantages with respect to [BK1] Theorem 0.1]: the bound $d \geq g-2 q+1$ is optimal, while in [BK1, Theorem 0.1], we only obtained $d \geq g-q$; the curve $C$ is arbitrary; the algebraically closed base field may have arbitrary characteristic; the bound $g \geq 4 q+5$ is usually better than the bound $g \geq 5 q+1$ used in BK1]. The weak point of Theorem 1.1 here with respect to [BK1, Theorem 0.1] is that $X$ is not an arbitrary smooth genus $g$ covering of $C$ and that for any such $X$ we allow here only one integer $d$. The bound in BK2. Theorem 1.1 and Proposition 1.5] is $d \geq g-q+1$, but we considered there a much harder problem about the irreducibility of $W_{d}^{1}(X)$.

For the proof of Theorem 1.1 we were motivated by the study of reducible curves, which are defined in the following way. Let $C$ be an integral projective curve. A reducible double covering of $C$ is a connected reduced projective curve $X$ with

Received by the editors January 15, 2001 and, in revised form, July 7, 2003.

2000 Mathematics Subject Classification. Primary 14H51, 14H30.

Key words and phrases. Double coverings, base-point-free pencil, Castelnuovo-Severi inequality, Brill-Noether theory.

The first named author was partially supported by MIUR and GNSAGA of INdAM (Italy). The second named author was supported by Korea Research Foundation Grant \#2001-015-DS0003.

(C)2004 American Mathematical Society 
exactly two irreducible components, say $X=X_{1} \cup X_{2}$, equipped with a morphism $f: X \rightarrow C$ such that $f_{\mid X_{1}}: X_{1} \rightarrow C$ and $f_{\mid X_{2}}: X_{2} \rightarrow C$ are isomorphisms. Alternatively, $X$ is uniquely determined by taking two curves, say $X_{1}$ and $X_{2}$, isomorphic to $C$, fixing isomorphisms $j_{1}: X_{1} \rightarrow C, j_{2}: X_{2} \rightarrow C$, a zero-dimensional subscheme $Z$ of $C, Z \neq \emptyset$, and fixing an isomorphism between $j_{1}^{-1}(Z)$ and $j_{2}^{-1}(Z)$. We have

$$
p_{a}(X)=2 p_{a}(C)+\operatorname{length}(Z)-1=2 p_{a}(C)+\operatorname{length}\left(X_{1} \cap X_{2}\right)-1,
$$

where $X_{1} \cap X_{2}$ is just seen as a zero-dimensional scheme.

\section{Reducible COVERINGS}

Let $f: X=X_{1} \cup X_{2} \rightarrow C$ be a reducible and connected double covering. If $C$ is smooth, then at each point of $X_{1} \cap X_{2}$ the double covering $X$ has two smooth branches and hence a planar singularity with multiplicity two (a node or a tacnode, perhaps a non-ordinary one). For every $M \in \operatorname{Pic}(X)$ we have $\operatorname{deg}(M)=\chi(M)-$ $\chi\left(\mathcal{O}_{X}\right) . M$ uniquely determines $M_{1}:=M_{\mid X_{1}} \in \operatorname{Pic}\left(X_{1}\right)$ and $M_{2}:=M_{\mid X_{2}} \in \operatorname{Pic}\left(X_{2}\right)$ and an isomorphism, $j$, between $M_{1 \mid X_{1} \cap X_{2}}$ and $M_{2 \mid X_{2} \cap X_{1}}$. Vice versa, at least if $X$ is nodal at each point of $\left(X_{1} \cap X_{2}\right)_{\text {red }}$, any such triple $\left(M_{1}, M_{2}, j\right)$ uniquely determines a line bundle on $X$. We have $\operatorname{deg}(M)=\operatorname{deg}\left(M_{1}\right)+\operatorname{deg}\left(M_{2}\right)$. If $C$ is smooth, it is easy to extend the classical Castelnuovo-Severi inequality to reducible connected double coverings of $C$.

Lemma 2.1. Let $C$ be a smooth curve and $f: X \rightarrow C$ a reducible and connected double covering. Set $g:=p_{a}(X)$ and $q:=p_{a}(C)$. Fix integers $d_{1}, d_{2}$ with $d_{1}>$ $0, d_{2}>0$ and $d_{1}+d_{2} \leq g-2 q$. Let $M \in \operatorname{Pic}(X)$ be a spanned line bundle such that $\operatorname{deg}\left(M_{\mid X_{1}}\right)=d_{1}$ and $\operatorname{deg}\left(M_{\mid X_{2}}\right)=d_{2}$. Then $d_{1}=d_{2}$, and there is an $R \in \operatorname{Pic}(C)$ with $\operatorname{deg}(R)=d_{1}, h^{0}(C, R)=h^{0}(X, M)$ and $M \cong f^{*}(R)$.

Proof. Since $d_{1}+d_{2} \neq 0$ and $M$ is spanned, we have $h^{0}(X, M) \geq 2$. Since $\operatorname{dim}(X)=$ 1, there is a linear subspace $V$ of $H^{0}(X, M)$ with $\operatorname{dim}(V)=2$ and $V$ spanning $M$. Fix any such $V . V$ induces a morphism $u: X \rightarrow \mathbb{P}^{1}$ with $\operatorname{deg}\left(u_{\mid X_{1}}\right)=d_{1}$ and $\operatorname{deg}\left(u_{\mid X_{2}}\right)=d_{2}$. The pair $(f, u)$ gives a morphism $h:=(f, u): X \rightarrow C \times \mathbb{P}^{1}$ such that $h\left(X_{i}\right)$ is an irreducible curve of type $\left(1, d_{i}\right)$ on the ruled surface $C \times \mathbb{P}^{1}$. Each curve $h\left(X_{i}\right)$ is isomorphic to $C$ because $f_{\mid X_{i}}$ is an isomorphism. If $h\left(X_{1}\right)=h\left(X_{2}\right)$, we obtain $d_{1}=d_{2}$, and, up to identification of $X_{1}$ with $h\left(X_{1}\right)$ given by $h$ and of $h\left(X_{1}\right)$ with $C$ obtained using the first projection of $C \times \mathbb{P}^{1}$, we may take as $R$ the line bundle $M_{\mid X_{1}}$. Hence we may assume $h\left(X_{1}\right) \neq h\left(X_{2}\right)$. Since the scheme $h\left(X_{1}\right) \cap h\left(X_{2}\right)$ is zero-dimensional, it has length $d_{1}+d_{2}$ by the intersection theory of the smooth ruled surface $C \times \mathbb{P}^{1}$. Hence $p_{a}(h(X))=2 q+d_{1}+d_{2}-1$. Since $p_{a}(h(X)) \geq p_{a}(X)=g$, we obtain $d_{1}+d_{2} \geq g-2 q+1$, as wanted.

Theorem 2.2. Fix integers $g, q, d_{1}$ and $d_{2}$ with $q \geq 0, d_{1} \geq q+3, d_{2} \geq q+$ $3, d_{1}+d_{2} \geq g-2 q+1$ and $g \geq 2 q$. Let $C$ be a smooth curve with $p_{a}(C)=q$. Then there exists a reducible and connected double covering $f: X=X_{1} \cup X_{2} \rightarrow C$ with $X$ a nodal curve, $p_{a}(X)=g$ and $M \in \operatorname{Pic}(X)$ with $\operatorname{deg}\left(M_{\mid X_{1}}\right)=d_{1}, \operatorname{deg}\left(M_{\mid X_{2}}\right)=$ $d_{2}, M$ spanned by its global sections and such that the morphism $X \rightarrow \mathbb{P}\left(H^{0}(X, M)\right)$ induced by $H^{0}(X, M)$ does not factor through $f$.

Proof. We have

$$
\operatorname{Pic}\left(C \times \mathbb{P}^{1}\right) \cong \operatorname{Pic}(C) \oplus \mathbb{Z}[F] \cong \operatorname{Pic}(C) \oplus \mathbb{Z}
$$


where $F$ will denote the class of a fiber of the projection $C \times \mathbb{P}^{1} \rightarrow \mathbb{P}^{1}$ onto the second factor. For any integer $a$, and any $M \in \operatorname{Pic}(C), M+a F$ will denote the corresponding class in $\operatorname{Pic}\left(C \times \mathbb{P}^{1}\right)$. We will often use the same additive notation for line bundles and divisors on $C \times \mathbb{P}^{1}$. By the Künneth formula for every $a \in$ $\mathbb{Z}$ and every $M \in \operatorname{Pic}(C)$ with $h^{0}(C, M)=0$, we have $h^{1}\left(C \times \mathbb{P}^{1}, M+a F\right)=$ 0 . By the Künneth formula for every $a<0$ and every $M \in \operatorname{Pic}(C)$, we have $h^{0}\left(C \times \mathbb{P}^{1}, M+a F\right)=0$. By the Künneth formula for every $a \geq 0$ and every $M \in$ $\operatorname{Pic}(C)$, we have $h^{0}\left(C \times \mathbb{P}^{1}, M+a F\right)=(a+1) h^{0}(C, M)$. Fix general $M_{i} \in \operatorname{Pic}(C)$ with $\operatorname{deg}\left(M_{i}\right)=d_{i}, i=1,2$. Hence $h^{1}\left(C, M_{i}\right)=0, h^{0}\left(C, M_{i}\right)=d_{i}+1-q$ and $M_{i}$ is very ample. Let $C_{i}$ be a general element of $\left|M_{i}+F\right|$. The very ampleness of $M_{i}$ implies that $\left|M_{i}+F\right|$ separates points and tangent vectors, and gives very easily, just by a dimension count (without even using any Bertini-type theorem), that each $C_{i}$ has no fiber of the first projection of $C \times \mathbb{P}^{1}$ as an irreducible component. Since $C_{i} \in\left|M_{i}+F\right|$, this implies that the map $C_{i} \rightarrow C$ induced by the first projection is an isomorphism. In particular, $C_{1}$ and $C_{2}$ are smooth. We also may assume $C_{1} \neq C_{2}$, and hence length $\left(C_{1} \cap C_{2}\right)=\left(M_{1}+F\right) \cdot\left(M_{2}+F\right)=d_{1}+d_{2}$. We claim that for a general pair $\left(C_{1}, C_{2}\right)$, the curve $C_{1} \cup C_{2}$ has only ordinary nodes as singularities. To check the claim we fix $C_{1}$ (any smooth $C_{1} \in\left|M_{1}+F\right|$ will work) and consider the linear system $\left|M_{2}+F\right|$. Since $M_{2}$ is very ample, it has no base point and it separates the tangent vectors. Hence by a characteristic free form of Bertini's theorem, the restriction of $\left|M_{2}+F\right|$ to $C_{1}$ is a linear system whose general member is smooth, i.e., formed by $d_{1}+d_{2}$ distinct points. Hence the claim, i.e., for general $C_{1}$ and $C_{2}$ the curve $C_{1} \cup C_{2}$ is nodal. Now we fix a subset $S$ of $C_{1} \cap C_{2}$ with $\operatorname{card}(S)=d_{1}+d_{2}-g+2 q-1$. By assumption we have $d_{1}+d_{2}-g+2 q-1 \geq 0$; if $d_{1}+d_{2}-g+2 q-1=0$ we take $S=\emptyset$. Let $\pi: X \rightarrow C_{1} \cup C_{2}$ be the partial normalization of $C_{1} \cup C_{2}$ in which we normalize exactly the nodes of $C_{1} \cup C_{2}$ that lie in $S$. Since $g \geq 2 q$ we have $d_{1}+d_{2}>\operatorname{card}(S)$, i.e., $X$ is a connected nodal curve with exactly two irreducible components and $p_{a}(X)=g$. Let $f: X \rightarrow C$ be the composition of $\pi$ with the morphism $C_{1} \cup C_{2} \rightarrow C$ induced by the projection $C \times \mathbb{P}^{1} \rightarrow C$. The pair $(X, f)$ is the connected reducible double covering of $C$ we were looking for.

\section{Proof of Theorem 1.1}

In this section we prove Theorem 1.1.

Proof of Theorem 1.1. The result is obvious if $d \geq g+1$; just take a general $M \in$ $\operatorname{Pic}^{d}(X)$ (which is non-special). Hence from now on we assume $d \leq g$. We will use the notation concerning $\operatorname{Pic}\left(C \times \mathbb{P}^{1}\right)$ introduced in the proof of Theorem 2.2. Let $\mathcal{O}$ be the structure sheaf of $C \times \mathbb{P}^{1}$. For any $U \in \operatorname{Pic}\left(C \times \mathbb{P}^{1}\right)$ and all finite subsets $S, S^{\prime}$ of $C \times \mathbb{P}^{1}$ with $S \cap S^{\prime}=\emptyset$, set

$$
|U|\left(-S-2 S^{\prime}\right):=\left\{D \in|U|: S \subset D \text { and } D \text { is singular at each point of } S^{\prime}\right\} .
$$

If $S=\left\{P_{1}, \cdots, P_{s}\right\}$ and $S^{\prime}=\left\{Q_{1}, \cdots, Q_{t}\right\}$, set

$$
|U|\left(-P_{1}-\cdots-P_{s}-2 Q_{1}-\cdots-2 Q_{t}\right):=|U|\left(-S-2 S^{\prime}\right) .
$$

The canonical line bundle of $C \times \mathbb{P}^{1}$ is $\omega_{C}-2 F$. Hence by the adjunction formula for every $A \in \operatorname{Pic}^{x}(C), x>0$, and every integer $b \geq 0$ with $|A+b F| \neq \emptyset$ and every $D \in|A+b F|$ we have $\omega_{D} \cong\left(A+\omega_{C}+(b-2) F\right)_{\mid D}$ and hence $p_{a}(D)=1+b x-x+b q-b$. In particular, for every $D \in|A+2 F|$ with $\operatorname{deg}(A)=d$ we have $p_{a}(D)=-1+d+2 q$. 
For any such $D$ we have $h^{1}\left(C \times \mathbb{P}^{1}, \mathcal{O}(-D)\right)=0$ (Künneth formula); here we use $x \neq 0$. Hence by the exact sequence

$$
0 \rightarrow \mathcal{O}(-D) \rightarrow \mathcal{O} \rightarrow \mathcal{O}_{D} \rightarrow 0
$$

we obtain $h^{0}\left(D, \mathcal{O}_{D}\right)=1$. In particular, $D$ is connected. First we will do the case $d=g-2 q+1$; by assumption we also have $d \geq 2 q+6$. Fix $A \in \operatorname{Pic}^{d}(C)$ and take a general $X \in|A+2 F|$.

Claim I. $X$ is a smooth connected curve of genus $g$.

Proof of Claim I. We just saw that $p_{a}(X)=g$ and that $X$ is connected. Since $d \geq 2 q+6$, we may find integers $d_{1}, d_{2}$ with $d_{1} \geq q+3, d_{2} \geq q+3$ and $d_{1}+d_{2}=d$. Hence, taking a general $M_{i} \in \operatorname{Pic}(C)$ with $\operatorname{deg}\left(M_{i}\right)=d_{i}$ and a general $C_{i} \in\left|M_{i}+F\right|$, we obtain a nodal curve $C_{1} \cup C_{2} \in\left|M_{1}+M_{2}+2 F\right|$ (proof of Theorem 2.2). Hence, taking $A=M_{1} \otimes M_{2}$, we know that $X$ has at most nodal singularities. However, since each linear system $\left|M_{i}+F\right|$ has no base points and separates tangent vectors, Bertini's theorem gives the smoothness of $X$ in arbitrary characteristic.

Notice that Claim I proves Theorem 1.1 for $d=g-2 q+1$, because every $X \in|A+2 F|$ comes with a morphism $X \rightarrow \mathbb{P}^{1}$ that does not factor through the double covering $X \rightarrow C$, which is the restriction to $X$ of the projection map $C \times \mathbb{P}^{1} \rightarrow C$. Now we assume $g \geq d>g-2 q+1$ (and again $d \geq 2 q+6$ ). Fix a general $A \in \operatorname{Pic}^{d}(C)$.

Claim II. There is $Y \in|A+2 F|$ with $Y$ integral and with exactly $d-g+2 q-1$ ordinary nodes as its only singularities.

Proof of Claim II. By Claim I, there is $B \in\left|A_{1}+2 F\right|$, $\operatorname{deg}\left(A_{1}\right)=g-2 q+1$ and $A_{1}$ general, with $B$ smooth. Since $\operatorname{dim}\left(\left|A_{1}+2 F\right|\right)=3(g-3 q+2)-1 \geq 3(q+7)>$ $2 q-1 \geq d-g+2 q-1$, for a general $S \subset C \times \mathbb{P}^{1}$ with $\operatorname{card}(S)=d-g+2 q-1$ we may find a smooth $B \in\left|A_{1}+2 F\right|$ with $S \subset B$. By the generality of $S$, the image of $S$ by the projection $u_{1}: C \times \mathbb{P}^{1} \rightarrow C$ is a set $S^{\prime}$ with $\operatorname{card}\left(S^{\prime}\right)=d-g+2 q-1$. Set $T:=B \cup u_{1}^{-1}\left(S^{\prime}\right)$. Since $S$ may be considered as a general subset of $B$, the curve $T$ is nodal. First assume $d=g-2 q+2$, i.e., $\operatorname{card}(S)=1$, say $S=\{P\}$. $T$ has two irreducible components and exactly two nodes. By the generality of $P$ we have $\operatorname{dim}(|A+2 F|(-P))=\operatorname{dim}(|A+2 F|)-1$. Furthermore, the linear system $|A+2 F|(-2 P)$ separates the tangent vectors at $P$. Here we may use that $A$ is very ample and that $|A+2 F|(-P)$ contains reducible elements, since

$$
\operatorname{dim}\left(\left|A\left(-u_{1}(P)\right)+2 F\right|\right)=\operatorname{dim}(|A+2 F|)-3
$$

and a general element $E$ of $\left|A\left(-u_{1}(P)\right)+2 F\right|$ does not contain $P$, i.e.,

$$
E \cup u_{1}^{-1}\left(u_{1}(P)\right) \notin|A+2 F|(-2 P) \text {. }
$$

Hence a general element $Y$ of $|A+2 F|(-2 P)$ does not have $u_{1}^{-1}\left(u_{1}(P)\right)$ as a component. Thus $Y$ must be irreducible and with exactly one node, as wanted. Now assume $d>g-2 q+2$, i.e., $\operatorname{card}(S) \geq 2$. Fix $P, Q \in S$ with $P \neq Q$ and set $T_{Q}:=B \cup u_{1}^{-1}\left(S^{\prime} \backslash\left\{u_{1}\left(Q_{1}\right)\right\}\right)$ and $T_{P}:=B \cup u_{1}^{-1}\left(S^{\prime} \backslash\left\{u_{1}(P)\right\}\right)$. By induction on $d$ we may assume that a general element $T(Q)$ (resp. $T(P))$ of $\left|T_{Q}\right|(-2(S \backslash\{Q\}))$ (resp. $\left|T_{P}\right|(-2(S \backslash\{P\}))$ is irreducible, nodal and $\operatorname{Sing}(T(Q))=S \backslash\{Q\}$ (resp. $\operatorname{Sing}(T(P))=S \backslash\{P\})$. Since $T(Q) \cup u_{1}^{-1}\left(u_{1}(Q)\right)$ and $T(P) \cup u_{1}^{-1}\left(u_{1}(P)\right)$ are both 
elements of $|T|(-2 S)$, we easily obtain that a general element of $|T|(-2 S)$ is irreducible, nodal and singular only along $S$. Hence we obtain Claim II for the given integer $d$.

Take $Y \in|A+2 F|$ with $Y$ integral and with exactly $d-g+2 q-1$ ordinary nodes as its only singularities, and let $u: X \rightarrow Y$ be the normalization. There is a degree two morphism $f: X \rightarrow C$ obtained by composing $u$ with the restriction to $Y$ of the projection $C \times \mathbb{P}^{1} \rightarrow C$. There is a degree $d$ morphism $v: X \rightarrow \mathbb{P}^{1}$ obtained by composing $u$ with the restriction to $Y$ of the projection $C \times \mathbb{P}^{1} \rightarrow \mathbb{P}^{1}$, proving Theorem 1.1 for the integer $d$.

It should be possible to use $[\underline{\mathrm{R}}$, and $[\mathrm{S}$, Theorem $7(\mathrm{i})]$ to give a proof of Theorem 1.1, and this is quite easy in the cases $d=g-2 q+1$ and $d=g-2 q+2$. We do not know if this approach may be used to obtain the same result for some genus $g$ with $2 q-1 \leq g \leq 4 q+4$. On the other hand, if the genus of $X$ is relatively higher with respect to the genus of $C$, say $g \geq 8 q+4$, we can prove the existence of a double covering $f: X \rightarrow C$ such that for every $d \geq g-2 q+1$, there is a spanned $M \in \operatorname{Pic}^{d}(X)$ such that the morphism $X \rightarrow \mathbb{P}\left(H^{0}(X, M)\right)$ induced by $M$ does not factor through $f$.

Theorem 3.1. Fix integers $q \geq 0$ and $g$ with $g \geq 8 q-4$. Let $C$ be a smooth projective curve of genus $q$ defined over an algebraically closed field of characteristic zero. Then there exists a smooth connected curve of genus $g$ that admits a surjective degree two morphism $f: X \rightarrow C$ having a spanned $M \in \operatorname{Pic}^{d}(X)$ for any $d \geq$ $g-2 q+1$ such that the morphism $X \rightarrow \mathbb{P}\left(H^{0}(X, M)\right)$ induced by $M$ does not factor through $f$.

We need the following lemma, which one can prove easily by using elementary results in Brill-Noether theory; here we adopt the conventions and notation used in $\mathrm{ACGH}$.

Lemma 3.2. Fix an integer $e \geq 1$. Let $X$ be a smooth curve of genus $g \geq 4 e-4$ defined over an algebraically closed field of characteristic zero. For an integer d, let $\Sigma_{d}^{1}$ be the union of components of $W_{d}^{1}(X)$ whose general element is base-point-free and complete. If $\Sigma_{g-e+1}^{1} \neq \emptyset$, then every component of $\Sigma_{g-e+1}^{1}$ has the expected dimension $\rho(g-e+1, g, 1)=g-2 e$. Furthermore, we have $\Sigma_{g-e+2}^{1} \neq \emptyset$.

Proof. Since it is assumed that $\Sigma_{g-e+1}^{1} \neq \emptyset$, any component of $\Sigma_{g-e+1}^{1}$ has dimension at least $\rho(g-e+1, g, 1)=g-2 e$. Suppose there exists a component $\Sigma \subset \Sigma_{g-e+1}^{1}$ such that $\operatorname{dim} \Sigma=n \geq g-2 e+1$, and take a general $L \in \Sigma$. By the base-point-free pencil trick and the description of the tangent space to the scheme $W_{d}^{r}(X)$ in general, we have

$$
\begin{aligned}
h^{0}\left(X, L^{2}\right) & =2(g-e+1)-g+1+h^{1}\left(X, L^{2}\right) \\
& =2(g-e+1)-g+1+\operatorname{ker} \mu_{0} \\
& \geq g-2 e+3+n-\rho(g-e+1, g, 1)=n+3,
\end{aligned}
$$

where $\mu_{0}: H^{0}(X, L) \otimes H^{0}\left(X, K L^{-1}\right) \rightarrow H^{0}(X, K)$ is the natural map given by multiplication of sections; cf. [ACGH, Proposition 4.2; page 189]. Therefore, it follows that

$$
g-2 e+1 \leq n \leq \operatorname{dim} W_{2 g-2 e+2}^{n+2}(X) \leq \operatorname{dim} W_{2 g-2 e+2}^{g-2 e+3}(X)=2 e-4,
$$


contrary to the assumption $g \geq 4 e-4$. This completes the proof of the first assertion of our lemma. Suppose now that $\Sigma_{g-e+2}^{1}=\emptyset$. Then, we have

$$
W_{g-e+2}^{1}(X)=\left[\Sigma_{g-e+1}^{1}+W_{1}(X)\right] \cup\left[W_{g-e}^{1}(X)+W_{2}(X)\right] .
$$

Since $\operatorname{dim}\left[\Sigma_{g-e+1}^{1}+W_{1}(X)\right]=\rho(g-e+1, g, 1)+1<\rho(g-e+2, g, 1)$, it follows that the closed locus $\Sigma_{g-e+1}^{1}+W_{1}(X)$ is contained in $W_{g-e}^{1}(X)+W_{2}(X)$. Note that a general element in the locus $\Sigma_{g-e+1}^{1}+W_{1}(X)$ is a complete pencil with only one base point, whereas a complete pencil in $W_{g-e}^{1}(X)+W_{2}(X)$ has at least two base points, which is an absurdity.

Proof of Theorem 3.1. In the proof of Theorem1.1, it was shown that there exists a double covering $f: X \rightarrow C$ that has a spanned $M \in \operatorname{Pic}^{g-2 q+1}(X)$ such that the morphism $X \rightarrow \mathbb{P}\left(H^{0}(X, M)\right)$ induced by $M$ does not factor through $f$. We will argue that for this double covering $f: X \rightarrow C$ and for every $d \geq g-2 q+1$, there also exists $M^{\prime} \in \operatorname{Pic}^{d}(X)$ such that the morphism $X \rightarrow \mathbb{P}\left(H^{0}\left(X, M^{\prime}\right)\right)$ induced by $M^{\prime}$ does not factor through $f$. We now take $e=2 q$ in Lemma 3.2 By Theorem 1.1, we have $\Sigma_{g-2 q+1}^{1} \neq \emptyset$ and hence $\Sigma_{g-2 q+2}^{1} \neq \emptyset$ by Lemma 3.2. By taking $e^{\prime}=2 q-1$ in Lemma 3.2 we again have $\Sigma_{g-e^{\prime}+2}^{1}=\Sigma_{g-2 q+3}^{1} \neq \emptyset$; note that $g \geq 8 q-4>4 e^{\prime}-4$. We may continue this process by taking smaller $e$ 's until $e=2$ and we stop.

\section{REFERENCES}

[ACGH] E. Arbarello, M. Cornalba, P. A. Griffiths, and J. Harris: Geometry of Algebraic Curves I, Springer-Verlag, New York, 1985. MR 86h:14019

[BK1] E. Ballico and C. Keem: On multiple coverings of irrational curves, Arch. Math. (Basel) 65 (1995), 151-160. MR 96g:14020

[BK2] E. Ballico and C. Keem: Variety of linear systems on double covering curves, J. Pure Appl. Algebra 128 (1998), 213-224. MR 99c:14042

[Ka] E. Kani: On Castelnuovo's equivalence defect, J. reine angew. Math. 352 (1984), 24-70. MR 86d:14029

[R] I. Reider: Vector bundles of rank 2 and linear systems on algebraic surfaces, Ann. Math. (2) 127 (1988), 309-316. MR 89e:14038

[S] N. I. Shepherd-Barron: Unstable vector bundles and linear systems on surfaces in characteristic p, Invent. Math. 106 (1991), 243-262. MR 92h:14027

Department of Mathematics, Università di Trento, 38050 Povo(TN), Italy

E-mail address: ballico@science.unitn.it

Department of Mathematics, Seoul National University, Seoul 151-742, South Korea

E-mail address: ckeem@math.snu.ac.kr

Department of Mathematics, Seoul National University, Seoul 151-742, South Korea E-mail address: s2park@math.snu.ac.kr

Current address: Mathematics Section, The Abdus Salam International Centre for Theoretical Physics, Strada Costiera 11, 34014 Trieste, Italy

E-mail address: spark@ictp.trieste.it 